\title{
Survey on Customized Tourism and Travel Recommendation System based on User-location Vector Approach
}

\author{
Aditi Mhadgut \\ BE Computer \\ MMCOE, Pune
}

\author{
Gauri Bhagwat \\ BE Computer \\ MMCOE, Pune
}

\author{
Pooja Dhule \\ Asst. Professor \\ MMCOE, Pune
}

\begin{abstract}
Location-based social networks provide people with an interface to share their locations and write reviews about interesting places of attraction. Social networking websites allows us to understand the user's interest and behavior pattern on various Travel and Tourism services, especially travel attractions and point of interest, which can be exploited to recommend personalized list of places to users. The major challenge faced by Travel and Tourism recommendation system is to understand the indirect relationships that exist between the user and the point of interest. In this paper, userlocation vector approach to represent and identify the implicit relationship between user and point of interest using a vector have been used.
\end{abstract}

\section{General Terms}

1. RS-Recommendation System

2. TRS- Tourist Recommendation System

3. ULV- User Location Vector

\section{Keywords}

Social media mining, Tourism, Recommendation System, User- Location vector

\section{INTRODUCTION}

Nowadays, tourism plays a vital role in nation's economic growth. Of which e-Tourism is a major part. e-Tourism is a tourist recommendation and planning application to assist users on the organization of a leisure and tourist agenda. eTourism makes recommendations based on the user's choice, his demographic classification, the places visited by the user in former trips and, finally, his current visit preferences.

A great number of services now exist that can offer support or help the traveler before, during and after the travel. There is a vast information related to travel destination and their associated resources such as Accommodation, restaurant, Museums, Transports or events available on the Internet. When the amount of online information and services increases, it becomes very complex and time consuming for tourist to discover the relevant information. Modern tourists are advanced not only by the desire to reduce costs, but also use of contextual information society in which the necessary information is available. Nowadays tourist trusts less in advertising brochures of travel agents, does not want to pay for expensive travel guides. At the planning stage of their journey tourist usually tends to make his own analysis for information with the help of large information portals, online feedback, photos, videos and comments submitted by those who have visited this trip. Nowadays the actual problem is not the traditional multi filtering search of relevant information, but search into be person oriented, personalized, adapted to the individual needs of particular "sophisticated" users need information. User's searches request for urgent information resources in extended web environment occasionally is faced with the problem of selection of useful data in this field. This Problem can be solved with the help of the Recommendation Engine. User generated data from the social media can be processed, and used by the recommendation systems. Recommender system is a field of Information Retrieval that are widely used in our day to day applications and ecommerce sites to filter product and services according to user interest and preferences. A recommendation system is a subclass of iinformation filtering system that helps to predict the "rating" or "preference" a user would give to an item.

\subsection{The Common Types of Recommendation Systems Available Today are \\ 1.1.1 Content Based Recommendation System}

It is content based recommendation system,when the data considered for the recommendation is user profile and their past history.

\subsubsection{Collaborative-Filtering Based \\ Recommendation System}

Collaborative filtering based recommendation system recommends using both history of the user under consideration and also the history of the users who are similar to considered user.

\subsubsection{Hybrid Recommendation System}

A hybrid recommendation system is the combination of the content based and collaborative filtering based recommendation system.

\section{LITERATURE SURVEY}

The major issue with the previous recommender is that it is not personalized for a particular user. Another major problem faced by the Travel and Tourism recommendation system is to find out the relationship between the places and the users[4]. To overcome the above problem, here the approach called user-location vector has been used which has a weighted value for places of interest that corresponds to the relationship that the user and the place of interest shares. This Recommender system analyses the user's profile, builds the user-location vector and recommends a ranked list of places totally based on the user's interest and preferences[1]. 


\section{ARCHITECTURE}

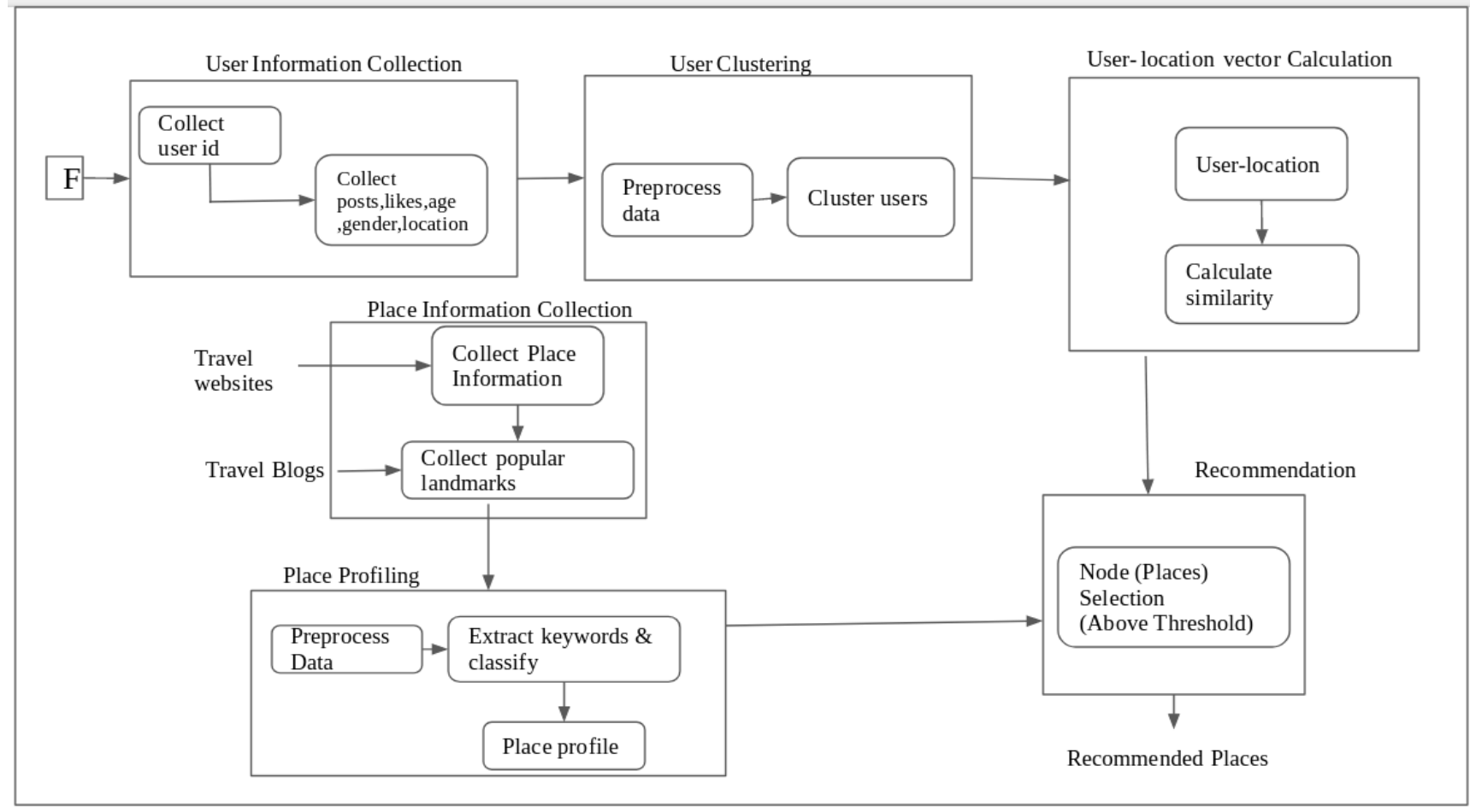

Fig 1: System Architecture

\section{ALGORITHMS}

\subsection{Algorithm1}

User Information Collection (UserId)

Input:User Id

Output: Characteristics and properties of user: age, tagged-locations, likes, posts, emotions, gender

Begin

If(UserId) then

return (age, tagged-locations, likes, posts,emotions, gender)

else

return NULL

End

\subsection{Algorithm2}

Data Preprocessing (Data)

Input:Data- tagged-locations, posts, emotions, likes, age, gender

Begin

if(Data) then

return data

End

Else if(!tagged-location or !posts or ! emotions or !likes) then

return -1 (discard data)

End

End

\subsection{Algorithm3}

K-Means Clustering(n,I,k,d,c)

Input: n-no. of users, i-information of users, k- no. of clusters,d-distance between centroids and users in clusters, c-cluster of users (assume k-centroids)

Begin while(d greater than 0.1) do

for $\mathrm{j}=1$ to $\mathrm{n}$ do

$\mathrm{d}$ is calculated with all centroids $\mathrm{i}$ is assigned to a cluster

end

Recompute centroid // mean of its user points

End

Return c

End

\subsection{Algorithm4}

User location vector calculation algorithm Input:N- number of users, v-number of tagged locations for each user, l-likes on locations, mnumber of point of interest, $\mathrm{j}$-number of tagged location for a user, k-number of likes on a location for a user $k$. Output : $\underline{\mathrm{ULV}}$ (User Location Vector)

Begin

for $\mathrm{j} 1$ to $\mathrm{N}$ do

for $\mathrm{p} 1$ to $\mathrm{m}$ do

ULV [j][p] Normalize $((2 * j[\mathrm{p}]+\mathrm{k}[\mathrm{p}]) /(\mathrm{v}+1))$;

end

end 
return $\underline{\mathrm{ULV}}$;

End

\subsection{Algorithm5}

Node Selection(s,i,t)

Input: s similar users, i user-place vector,t threshold

Output: $p$ place of interest of the user

Begin

for $j=1$ to $s$ do if(i[j] greater than $t)$ then

P places retrieved from ontology

return $p$

End

else

return null

end

end

End

\subsection{Algorithm6}

User similarity computation(g,n)

Input: $\mathrm{g}$ - user to be compared, $\mathrm{n}$ is the number of users

Output: User Similarity calculated based on Euclidean distance measure

Begin

$\mathrm{s}=0$;

for $\mathrm{j}=1$ to $n$ do

for $\mathrm{k}=1$ to $\mathrm{m}$ do

$\mathrm{s}=\mathrm{s}+\underline{\operatorname{pow}}((\mathrm{g}[\mathrm{k}]-\mathrm{i}[\mathrm{k}]), 2)$;

End

$\operatorname{dis}=1 / \operatorname{sqrt}(\mathrm{s})$;

end

End

\subsection{Algorithm7}

Ranking(p,i)

Input: p-no. osf selected places, i- user- place vector

Output: $r$-sort places based on the vector

Begin for $\mathrm{j}=1$ to $\mathrm{p}$ do

$\mathrm{r}=$ sort places based on the user-location vector ranked based on

True Bayesian Estimator

end

return $\mathrm{r}$

End

\section{MERITS}

[1] A hybrid approach combines the two types of information,while it is also possible to use the recommendations of the two filtering techniques independently.

[2] Using the user-location vector, the recommendation system predicts the user's interest more accurate that other existing system.

\section{DEMERITS}

[1] The limitations include going through the list of places in the place profile while recommending which is ineffective use of time.

\section{CONCLUSION AND FUTURE WORK}

Nowadays, Recommendation system is base for providing needful and accurate data to the users, in the world of internet. The use of hybrid recommendation system gives the better result than by either using content based recommendation system or collaborative filtering recommendation system. Hybrid recommendation system has several advantages like its more personalized, dynamic and efficient. It can be used in several domains like e-commerce, e-tourism, medical, media,etc. E-tourism RSs are getting popular day by day. Thus TRSs which uses user location vector,predicts the users interest more accurate than that of other RSs. In order to overcome the limitations, ontology of places can be constructed. Another future enhancement can be to recommend a travel plan using the recommended places.

\section{REFERENCES}

[1] IEEE paper-"A user-location vector based approach for personalised tourism and travel recommendation " by Ajantha D,Jobi Vijay and Raji Sridhar,2017.

[2] Zhiwen $\mathrm{Yu}$, Huang $\mathrm{Xu}$, Zhe Yang, and Bin Guo ,Personalized Travel Package With Multi-Point-of Interest Recommendation Based on Crowdsourced User Footprints, IEEE Transactions On Human-machine Systems, Vol. 46, No. 1,pp. 151 - 158, February 2016.

[3] Paper-"Personalized Dynamic Recommendation System for Tourism Using Hybrid Approach in Web Mining" by Priyanka B. Tiwari, 2Krunal J. Panchal published in International Journal of Engineering Development and Research.

[4] IEEE paper-"A Survey on Recommender Systems" by Motlatsi Liphoto,Chunling Du, Seleman Ngwira,2014.

[5] Kavitha S, Jobi Vijay and Rajeswari Sridhar (2017). 'Tourism Recommendation Using Social Media', Advance in Intelligent Systems and Computing, Proceeding of ICAIECES 2016 Artificial Intelligence and Evolutionary Computations in Engineering Systems, Volume. 517. Article in Press.

[6] Jonghyun Hana , Hyunju Lee b,'Adaptive landmark recommendations for travel planning: Personalizing and clustering landmarks using geo-tagged social media', Journal of Pervasive and Mobile Computing, Vol.18, Issue C,pp. 4-17,April 2015.

[7] Hsiu-Sen Chiang, Tien-Chi Huang,' User-adapted travel planning system for personalized schedule recommendation', Journal of Information Fusion,Vol.21,pp.3-17, January 2015. 